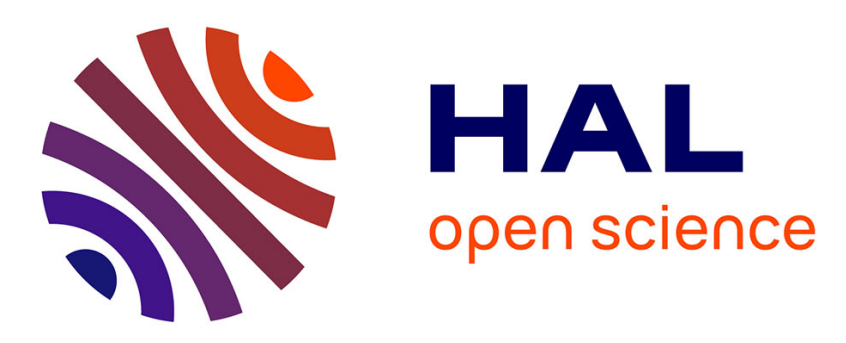

\title{
Rollback reconstruction for TDC enhanced perfusion imaging
}

\author{
Jia-Shun Liu, Yi-Kun Zhang, Hui Tang, Li-Bo Zhang, Ben-Qiang Yang, Ying \\ Yan, Li-Min Luo, Yang Chen
}

\section{- To cite this version:}

Jia-Shun Liu, Yi-Kun Zhang, Hui Tang, Li-Bo Zhang, Ben-Qiang Yang, et al.. Rollback reconstruction for TDC enhanced perfusion imaging. Nuclear science and techniques / Chinese Nuclear Society, 2021, 32 (8), pp.80. 10.1007/s41365-021-00918-7 . hal-03329632

\section{HAL Id: hal-03329632 https://hal.science/hal-03329632}

Submitted on 16 Sep 2021

HAL is a multi-disciplinary open access archive for the deposit and dissemination of scientific research documents, whether they are published or not. The documents may come from teaching and research institutions in France or abroad, or from public or private research centers.
L'archive ouverte pluridisciplinaire HAL, est destinée au dépôt et à la diffusion de documents scientifiques de niveau recherche, publiés ou non, émanant des établissements d'enseignement et de recherche français ou étrangers, des laboratoires publics ou privés. 


\title{
Rollback Reconstruction for TDC Enhanced Perfusion Imaging*
}

\author{
Jia-Shun Liu, ${ }^{1}$ Yi-Kun Zhang, ${ }^{1}$ Hui Tang, ${ }^{1}$ Li-bo Zhang, ${ }^{2}$ Ben-qiang Yang, ${ }^{2}$ Ying Yan, ${ }^{3}$ Li-Min Luo, ${ }^{1,4}$ and Yang Chen ${ }^{1,4}, \dagger$ \\ ${ }^{1}$ Laboratory of Image Science and Technology, Southeast University, Nanjing 210096, China \\ ${ }^{2}$ Department of Radiology, General Hospital of the Northern Theater of the Chinese People's Liberation Army, Shenyang 110016, China. \\ ${ }^{3}$ Department of Radiotherapy, General Hospital of the Northern Theater of the Chinese People's Liberation Army, Shenyang 110016, China. \\ ${ }^{4}$ Centre de Recherche en Information Biomedicale Sino-Francais (LIA CRIBs), Rennes 35000, France
}

\begin{abstract}
Tomographic perfusion imaging is a significant function imaging modality for stroke diagnosis. However, the low rotational speed of $\mathrm{C}$-arm is a challenge for the application of perfusion imaging in C-arm CBCT(from 6 to 8 seconds per circle). The traditional reconstruction methods can not remove the artifacts caused by the slow rotational speed or can not acquire enough sample points to restore the time density curve(TDC). This paper presents a dynamic rollback reconstruction method for CBCT. The proposed method could improve the temporal resolution by increasing the sample points used for calculating the TDC. Combined with of the existing research results, the algorithm allows the possibility of using slow rotating scanners for perfusion imaging purposes. In experiments, the proposed method is compared with other dynamic reconstruction algorithms which is based on standard reconstruction, temporal interpolation approach. The presented algorithm could improve the temporal resolution without increasing $\mathrm{X}$-ray exposure time or contrast agent.
\end{abstract}

Keywords: Rollback reconstruction, CBCT, Time resolution, Time density curve

\section{INTRODUCTION}

2 As one of leading causes of death, stroke may bring se3 rious long-term disability. According to the statistics, there 4 are about 2.5 million new stroke cases in China every year, 5 and about 1.7 million patients die from stroke. In acute 6 stroke treatment, time is life. Perfusion CT is a well-accepted 7 method for stroke diagnosis[1-3] whose aim is to assess the 8 blood supply of the tissue which has already found its way 9 into clinical routine. Together with perfusion MRI(magnetic oresonance imaging), they represent the primary imaging tech1 niques for patients with symptoms of stroke. After inject2 ing the contrast agent, the tissue was scanned multiple times 3 in succession to obtain the TDC[4-6] of each slice. The 14 curve reflects the changes of contrast agent concentration in 5 tissues[7-9], i.e. the changes of blood supply in tissues. ${ }_{6}$ Hence, perfusion CT can be used to identify tissue regions 7 that can be salvageable and may contribute to stroke therapy. ${ }_{18}$ However, there are still some challenges for perfusion CT. 9 One limitation is that perfusion CT could only produce func20 tional maps of a limited number of slices. CBCT(Cone Bean $\left.{ }_{21} \mathrm{CT}\right)[10,11]$ can be used to tackle the problem. The applica22 tion of large-area detectors allows users to conduct perfusion ${ }_{3}$ CBCT studies on the three-dimensional regions of interest. 24 However, there are two problems to be addressed.

The first is known as the temporal resolution problem[12]. 26 In order to reconstruct an image at any time $t$, we need for ${ }_{27}$ each projection at projection angle $\alpha$. However, the source

* This work was supported in part by the Postgraduate Research \& Practice Innovation Program of Jiangsu Province under Grant KYCX17 0104; in part by the China Scholarship Council under Grant 201706090248; in part by the State's Key Project of Research and Development Plan under Grants 2017YFC0109202, 2017YFA0104302 and 2018YFA0704102; in part by the National Natural Science Foundation under Grants 61871117 , 61828101, and 31571001; and in part by the Science and Technology Program of Guangdong under Grant 2018B030333001.

† Yang Chen, chenyang.list@ seu.edu.cn
28 turns with a restricted rotational speed $\omega$. When the speed $29 \omega$ is slow, it means the contrast agent attenuation during the 30 scanning process cannot be ignored. The scanning time of ${ }_{31}$ traditional perfusion CT is limited to two seconds per circle. 32 In such a short time, the attenuation of the contrast agent is 33 negligible. So direct reconstruction will not influence the final 34 result, i.e., the recovery of the time density curve. However, 35 the scanning time of CBCT is from 6 to 8 seconds per circle, 36 which is much longer than the traditional perfusion CT. The 37 concentration of the contrast agent in blood has a great in38 fluence on reconstruction quality. For example, it may result 39 in the artifacts or inaccurate values in the reconstructed im40 ages. This will directly influence the subsequent production ${ }_{41}$ of perfusion parameter maps. The second problem is called 42 the time sampling problem. After injecting the contrast agent, 43 the acquisition time of traditional perfusion CT and CBCT is 44 almost equal. However, due to the different rotational speed ${ }_{45} \omega$, the total number of CBCT perfusion is much less than tra46 ditional perfusion $\mathrm{CT}$. That leads to a lack of sampling points. ${ }_{47}$ When using the same interpolation method to fit the time den48 sity curve, fewer sample points will lead to inaccurate results. 49 The degraded time density curve will mislead the assessment 50 of the patient's condition.

51 Various strategies have been proposed to tackle the above 52 problems. Grangeat et al.[13] proposed a more sophisticated 53 approach for dynamic reconstruction compared to the tra54 ditional FDK algorithm[14, 15]. The dynamic reconstruc55 tion algorithm is based on short-scan reconstruction with the 56 tent Feldkamp(T-FDK) algorithm[16]. First, the cone-beam 57 projections, were rearranged into the fan-parallel beam pro58 jections. Second, the filtering operation was performed for 59 the projection data. Then the back-projection calculation 60 was divided into three parts, and each part covered 60 de61 grees. These incomplete back-projections were denoted as 62 partial block backprojections (PBBs). As a consequence of 63 the dynamic acquisition scheme, the values of each PBB were ${ }_{64}$ known every half-rotation. Hence, for every PBB, a time se${ }_{65}$ ries of values observed every $t_{\pi}$. In order to obtain the re- 
66 construction result at a certain time point, Grangeat and co67 workers performed linear regression on the closest $\mathrm{k}$ values 68 of the time series. This method was called k-mode linear re69 gression. K-mode linear regression effectively reduced the 70 impact of the temporal resolution problem, but did not solve 71 the time sampling problem: it limited the attenuation of the 72 contrast agent during the scanning process from the original 73 to the current, but the number of sample points could not been ${ }_{129}$ 74 improved. Although a sample point could be obtained every $75 t_{\pi}$ by the rearrangement method, it was still not enough. Un76 der the CBCT scanning protocol, only a dozen sample points 77 could be obtained. At present, there is no good way to solve 78 the time sampling problem. From the perspective of data col79 lection, Fieselmann[17] proposed a method: when collecting 80 data, the patient was injected with the contrast agent twice. ${ }_{81}$ After the second injection of the contrast agent, a time offset 82 was added and data acquisition was not performed immedi83 ately. This time offset should be half the time of a single ${ }_{84}$ scan. In this way, the time sample points obtained are dou85 bled. However, this method has great drawbacks. First, it 86 did not solve the temporal resolution problem. More impor87 tantly, the contrast agent is harmful to human body, especially 88 the liver and kidneys, and some patients may also have al89 lergic reactions. Therefore, multiple injections of the con9o trast agent is obviously not recommended. In addition, the 91 author did not prove whether the first injection of contrast 92 agent will be left in the patient's body and whether it could 93 influence the results of the second injection of the contrast 94 agent. Jie Tang[18] proposed a temporal recovery method to 95 recover time density curves to enable $\mathrm{C}$-arm CBCT perfusion 96 studies. This method did not increase the number of sam97 ple points, but proposed a more excellent fitting method. In 98 their method, two optimization methods (CG and Bregman) 99 were used to solve the proposed temporal recovery problem. 100 Yinsheng Li[19] introduced a new technique referred to as en101 hanced SMART-RECON(eSMART-RECON) to enhance the 102 temporal performance in a multi-sweep CBCT data acquisi103 tion protocol. However, this method is an iterative recon104 struction method, which consumes a significant amount of 105 computing resources. In addition to the traditional methods, 106 with the rise of deep learning and neural network, it has also 107 been combined with perfusion. For example, Zhu[20] pro108 posed an optional way to reduce CTP imaging radiation dose, 109 that is downsampling 30-pass images to 15 passes in tempo110 ral domain and then restoring them to 30 passes with the deep 111 residual CNN model. It was also a method of recovering sam112 ple points. However, deep learning and neural network need 113 a lot of training data to ensure the accuracy of the results, and 114 the anti-risk ability is poor. If a little interference is added, 115 the result of the network may be wrong.

116 In this article, we address the problem of dynamic recon117 struction within the context of perfusion CT and presented 118 a dynamic rollback reconstruction approach for perfusion ${ }_{119}$ CBCT to improve the time density curve. And combining it 120 with the existing reconstruction method, it retains the advan121 tages of the original method. We took the contrast agent at- 17 122 tenuation caused by its propagation into account and assume 176 123 that there was no movement or deformation during the scan-

124 ning process. Then the temporal interpolation performed on 125 the partial block back-projections was employed to increase 126 the number of sampling points. Experiments demonstrated 127 the promising performance of the proposed rollback recon128 struction algorithm. 129 130

$3 \mathrm{D}$ reconstruction from $2 \mathrm{D}$ projections obtained along a 132 single circular source trajectory is most commonly calculated 133 using the FDK algorithm. However, the FDK algorithm can 134 not well preserve the reconstruction accuracy when the cone 135 angle is large. In 2000, Grass et al. proposed an alternative 136 approach based on a cone-beam to parallel-beam rebinning 137 step, a corresponding rebining step into a rectangular virtual 138 detector plane and a filtered backprojection. The computa139 tional complexity was lower than in Feldkamp's original ap140 proach. As shown in Fig.1, the fan-beam data acquired along 141 a circular source-detector trajectory can be rearranged into 142 sets of fans of rays. Similar to the algorithm of rearranging 143 fan-beam into parallel-beam, we can rearrange cone-beams 144 X-ray emitted from different positions of the ray source's cir145 cular trajectory into parallel-beams with the same cone angle 146 and parallel to each other in space. Although cone-beams X147 ray and parallel-beams X-ray are not completely correspond148 ing, they can be corresponded one by one by interpolation.

The X-ray source moves along a circular trajectory of ra150 dius $\mathrm{R}$, and the cone-beam projection datas $P_{\text {cone }}(\beta, m, n)$ at 51 various angles $\beta$ are collected on the flat panel detector. Then 152 these projection data are rearranged into the parallel-beam 153 projections, which are used to reconstruct the tomographic 154 images of the object. As shown in Fig.2, the ray received by 55 virtual detector 1 can be converted to a corresponding ray on 56 virtual detector 2 . The projection of the rearranged parallel57 beam on the X-Y plane is perpendicular to the virtual detector 58 2. The projection value of the rearranged parallel-beam on the 59 virtual detector 2 can be expressed by $P_{\text {para }}(\theta, g, s)$, where $\theta$ 60 is the projection angle of the parallel beam, $g$ and $s$ are the 161 horizontal and vertical coordinates of the rearranged paral162 lel beam on the virtual detector 2 , respectively. The specific 163 process can be divided into three steps: data rearrangement, 64 weighted filtering, and back-projection reconstruction.

(1)Data rearrangement

First, the cone-beam projections $P_{\text {cone }}(\beta, m, n)$ are rear${ }_{167}$ ranged into parallel-beam projection data $P_{\text {para }}(\theta, g, s)$. Dur168 ing transforming the $(\beta, m, n)$ space to $(\theta, g, s)$ space, not 169 every parallel-beam projection has the corresponding cone170 beam projection as the detector collected data are discrete. 171 Usually, the nearest interpolation or linear interpolation are 172 employed to tackle the above problem. Different interpola173 tion methods will influence the spatial resolution of the re74 constructed volume.

(2)Weighted filtering

Then, the rearranged parallel-beam projection data ${ }_{177} P_{\text {para }}(\theta, g, s)$ is weighted and filtered according to the fol- 


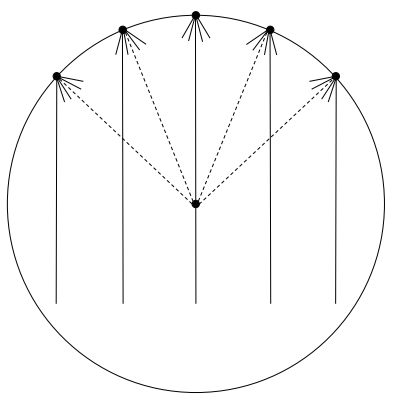

Fig. 1. The fan beam can be rearranged into a parallel beam.

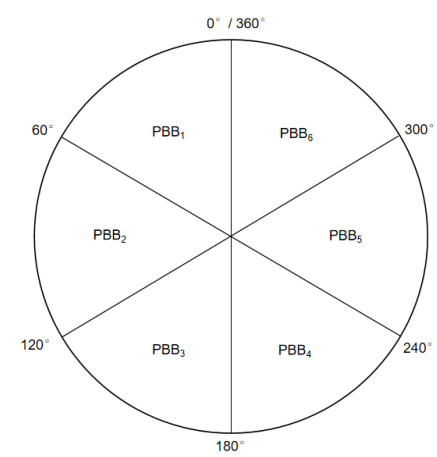

Fig. 3. Partial block back-projection.

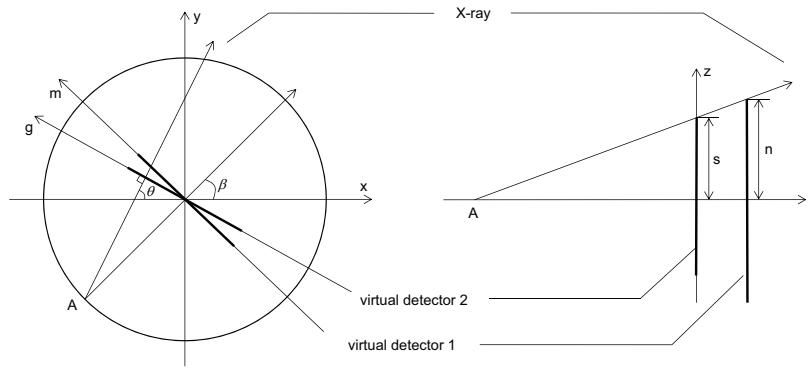

Fig. 2. Transform the $(\beta, m, n)$ space to $(\theta, g, s)$ space.

178 lowing formula to obtain $\tilde{P}_{\text {para }}(\theta, g, s)$ :

197 be tackled from the algorithm perspective. Pau et al. in198 troduced the block reconstruction strategy and presented the 199 TIA-TFDK(temporal interpolation approach, TIA) algorithm 200 to address the temporal resolution problem. First, the cone201 beam projections were rearranged into the parallel-beam pro202 jections: $P_{\text {cone }}(\beta, m, n) \rightarrow P_{\text {para }}(\theta, g, s)$. Then Pau et al. 203 introduced the $k \pi$-mode linear regression to reduce the im204 pact of temporal resolution problem. As shown in Fig.3. The 205 partial block back-projection operations were performed at 206 angular intervals of $2 \pi / N$ :

$$
\begin{array}{r}
P B B_{j}\left(X, t_{\frac{\pi}{N}(2 j+1)}\right)=\frac{1}{2} \int_{j \cdot \frac{2 \pi}{N}}^{(j+1) \cdot \frac{2 \pi}{N}} \tilde{P}_{\text {para }}(\theta, g, s) \mathrm{d} \theta \\
j=0,1, \ldots, N-1
\end{array}
$$
$\int \tilde{P}_{\text {para }}(\theta, g, s) \mathrm{d} \theta=\left[\sqrt{\frac{R^{2}-g^{2}}{R^{2}-g^{2}+s^{2}}} \cdot P_{\text {para }}(\theta, g, s)\right] * h\left(g_{209}^{208} \quad \begin{array}{l}\text { Using the general time interpolation method[21], each } \\ \text { block calculated independently of other blocks. The in- }\end{array}\right.$

179

180 where $\sqrt{\frac{R^{2}-g^{2}}{R^{2}-g^{2}+s^{2}}}$ represents the cosine value of the beam 181 cone angle $\kappa$ and $h(g)$ is the filter function. It is necessary 182 to multiply the projection data by the cosine weight since ${ }_{183} \tilde{P}_{\text {para }}(\theta, g, s)$ are the parallel-beam projections.

184 (3)Back projection reconstruction

185 Finally, the voxel $(x, y, z)$ is reconstructed using the cor-

${ }_{186}$ responding filtered parallel-beam projections $\tilde{P}_{\text {para }}(\theta, g, s)$.

187 The tomographic images can be obtained after all the voxels 188 are reconstructed. This process can be formulated as follows: 189

${ }_{190} F(x, y, z)=\int_{0}^{2 \pi} \tilde{P}_{\text {para }}(\theta, g(x, y, z), s(x, y, z)) \mathrm{d} \theta$

\section{B. TIA-TFDK algorithm}

As mentioned above, the contrast agent attenuation will 224 (2) 223 process was as follows:

$$
\begin{aligned}
H_{3}(x) & =\left[\left(1+2 \frac{x-x_{0}}{x_{1}-x_{0}}\right) y_{0}+\left(x-x_{0}\right) y_{0}^{\prime}\right]\left(\frac{x-x_{1}}{x_{0}-x_{1}}\right)^{2} \\
& +\left[\left(1+2 \frac{x-x_{1}}{x_{0}-x_{1}}\right) y_{1}+\left(x-x_{1}\right) y_{1}^{\prime}\right]\left(\frac{x-x_{0}}{x_{1}-x_{0}}\right)^{2}
\end{aligned}
$$

${ }_{193}$ lead to the temporal resolution problem when the scanning 225 Where $x_{0}$ and $x_{1}$ was the positions of two adjacent points 194 program consumes too much time. The best solution is 226 of the point to be interpolated, $y_{0}$ and $y_{1}$ correspond to the 195 to reduce the scanning time, but when the hardware condi- 227 dependent variables of $x_{0}$ and $x_{1}$, and $y_{0}^{\prime}$ and $y_{1}^{\prime}$ was the cor196 tions can not fulfil the requirement, the problem needs to 228 responding derivatives. The steps of interpolation of PBBs 
229 was summarized as follows:

$$
\begin{array}{r}
P B B_{j}(X, t)=H_{3}\left(P B B_{j}\left(X, t_{\frac{\pi}{N}(2 j+1)}\right)\right) \\
j=0,1, \ldots, N-1
\end{array}
$$

278 represents the time point of the scanning of $0-\pi$ and $t_{3 \pi / 2}$ 279 represents the time point of the scanning of $\pi-2 \pi$. There280 fore, after a single full scan, we can obtain the reconstructed 281 images at two time points: $t_{\pi / 2}$ and $t_{3 \pi / 2}$. This dynamic 282 acquisition process can be interpreted as sampling the time283 dependent projection $P_{t}$ with a period of $T_{s}=T_{\pi}$. These 284 sampling can be used to plot the density curve. Ideally, the 285 time density curve will be closer to the reference dynamic 286 curve as the number of sampling points increases. There287 fore, we propose the rollback reconstruction method to in288 crease the sampling points while not increasing the radiation 235

$$
F(x, y, z)=\sum_{j=0}^{N-1} P B B_{j}(X, t)
$$
290 reusing the projection data have been used for reconstruction. 291 The following steps have explained the rollback reconstruc292 tion method in detail.

${ }_{236}$ The partial block back-projection approach reduced the data 237 inconsistency in the projection domain. Even though the pro238 jections in a block were acquired at different times, the prob239 lem of time resolution was mitigated since the time between 240 angular intervals are shorter than the time of a short-scan re241 construction. The linear regression estimation had compen242 sated for the temporal evolution. In the experiments, Pau et al. ${ }^{207}$ 243 also proved that the data inconsistency would be significantly 298 244 reduced as $\mathrm{N}$ increased. But the calculation cost would also 245 increase synchronously. The TIA-TFDK algorithm is equiv246 alent to the FDK algorithm at $\mathrm{N}=1$. Furthermore, Pau et al. 247 concluded that the reconstruction quality and time could be 248 well balanced when $\mathrm{N}=6$.

\section{Rollback reconstruction}

250 The purpose of perfusion CBCT is to obtain an image se251 quence based on projection data acquired in several full ro252 tations. However, the time sampling problem may lead to 253 severe degradation of the time density curve. We adopt the 254 rollback reconstruction strategy to address this problem. The 255 cone-beam projection $P_{t}$ is indexed by the time t. The source 256 rotates at a constant angular speed $\omega$ from time $t=0$. The ac257 quisition could be performed either in continuous mode (the $258 \mathrm{X}$-ray source is always on) or in discontinuous mode (the $\mathrm{X}$ 259 ray source is switched off regularly). We assume that the 260 region of interest is within the FOV (field of view) of the 301 261 cylindrical detector. The projections at all angles are needed 302 262 to reconstruct the image frame at any time $t$. However, the 263 sampling points are discrete in practice. Thus, at the time ${ }_{264} t_{\alpha}$, we only could acquire the projection at the angular po265 sition $\alpha$. The same projection can be acquired again at time 30 ${ }_{266} t_{\alpha}+T_{2 \pi}$ after the source has finished a full rotation. Hence, 267 at the angular position $\alpha$, we could obtain a discrete sequence ${ }_{268} P_{t_{\alpha}}=P_{t_{\alpha}+T_{2 \pi}}$. This dynamic acquisition process can be in269 terpreted as sampling the time-dependent projection $P_{t}$ with 270 a period of $T_{s}=T_{2 \pi}$.

271 Since the cone-beam projections have been rearranged into 272 parallel-beam projections, the $180^{\circ}$ reconstruction condition 273 is extanded from the $2 \mathrm{D}$ to the $3 \mathrm{D}$ case. Hence, we can get 312 274 two reconstruction results after a full scan process. The one is 275 reconstructed using the projection data of $0-\pi$ and the other ${ }_{313}$ 276 is reconstructed with the projection data of $\pi-2 \pi$. Here we ${ }_{31}$ 277 take the middle time as the reference time point, that is, $t_{\pi / 2} 315$ the perfusion CBCT, as shown in Fig.5. This approach could
Start: $j=0$

(1)Starting from $0^{\circ}$, reconstruct the angle range from $0+$ $295 j \cdot \frac{\pi}{3}$ to $\pi+j \cdot \frac{\pi}{3}$, and record the result as $t_{\frac{\pi}{2}}+j \cdot \frac{\pi}{3}$

(2)Rollback $\frac{2 \pi}{3}$, in other words, $j=j+1$

(3)Repeat steps 1 and 2;

It can be expressed as:

$$
F_{\frac{\pi}{2}+j \cdot \frac{\pi}{3}}=\int_{0+j \cdot \frac{\pi}{3}}^{\pi+j \cdot \frac{\pi}{3}} \tilde{P}_{\text {para }}(\theta, g, s) \mathrm{d} \theta
$$

300 The number of reconstructed images will increase as the

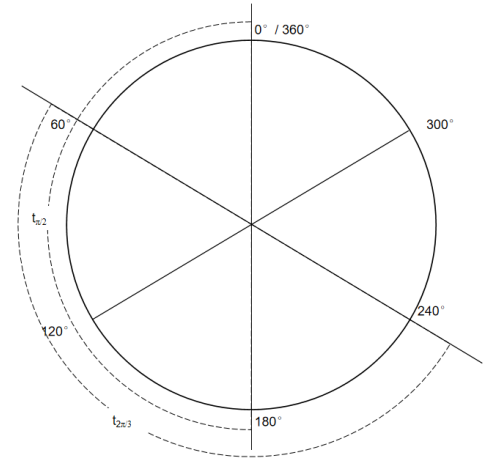

Fig. 4. Rollback reconstruction.

03 rollback angle increases. Hence, the presented rollback re304 construction could better preserve the accuracy of the time 305 density curve compared with the traditional reconstruction 6 method. Of course, the rollback reconstruction will increase 307 the computational cost because of the increasing of recon08 struction operation. Rollback reconstruction increases the 09 number of reconstruction sample points by reusing projection 310 data, and solves the time sampling problem, but it can't solve 311 the problem of time resolution.

\section{Rollback reconstruction with TIA-TFDK}

Combining the rollback reconstruction and TIA-TFDK algorithm, we propose an improved reconstruction method for 


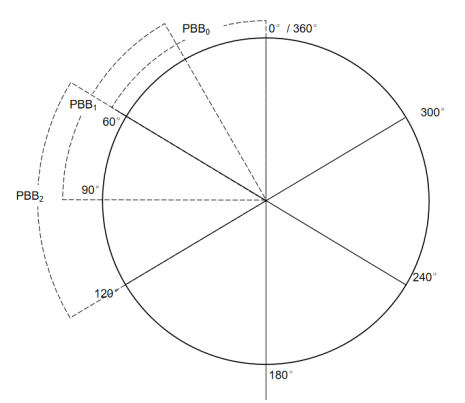

Fig. 5. Rollback reconstruction with TIA-TFDK.

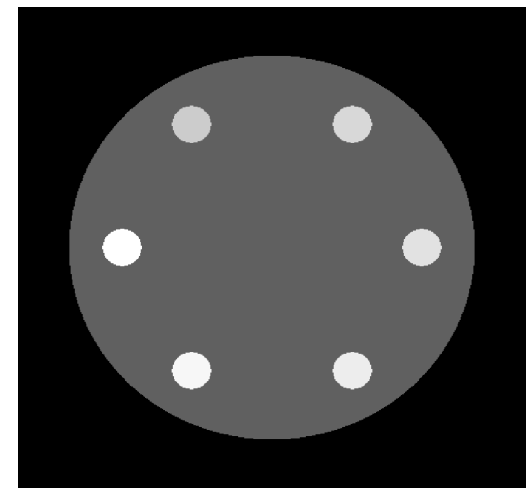

Fig. 6. Phantom A.

316 improve the reconstruction quality because it is promising 317 to tackle the temporal resolution problem and time sampling 318 problem simultaneously. The following steps explain the im319 proved rollback reconstruction method in detail.

(1)Rebinning cone-beam projections to parallel-beam pro321 jections:

322

323 324 PBBs with T-FDK:

325

$$
\begin{array}{r}
P B B_{j}=\int_{0+j \cdot \frac{\pi}{6}}^{\frac{\pi}{3}+j \cdot \frac{\pi}{6}} \tilde{P}_{\text {para }}(\theta, g, s) \mathrm{d} \theta \\
j=0,1, \ldots
\end{array}
$$
27 PBBs;

337 two reconstruction results. Furthermore, the number of sam-

338 pling points doubled after introducing the rollback method,

339 the optimization effect of TIA-TFDK algorithm on the time

340 resolution problem is retained. Thus, the accuracy of the 377

341 time density curve obtained by interpolation would be well-

343 preserved.

\section{E. Data preparation}

345 In this study, the CTP images were collected from eight 346 eligible slice locations of acute stroke patients. The scan pro8) 347 tocol is detailed as follows: tube voltage, $80 \mathrm{kV}$; tube current, $348250 \mathrm{~mA}$; slice thickness, $5 \mathrm{~mm}$. Each slice location has 30 349 CTP images with $512 \times 512$ pixels corresponding to 30 passes 350 in time sequence. Based on CTP, we refer to the scanning 351 protocol of CBCT, which reduces the number of samples ob352 tained in time sequence under the same scanning time. This 353 is due to the slower scanning speed of CBCT. We simulate 354 the scanning process of 8 seconds acquisition cycle, that is, 355 the time to obtain a reconstruction sample point is 8 seconds. 356 The original $512 \times 512 \times 30$ data volume for each slice loca357 tion was downsampled to the dataset of size $512 \times 512 \times 7$. 358 The detailed steps was as follows:

10) 359 (1)Through interpolation, expand the data $512 \times 512 \times 30$ 360 with a sampling period of 2 seconds into $512 \times 512 \times 300$ 361 with a sampling period of 0.2 seconds;

362 (2) Perform projection calculations. Since the experiment 363 simulates the CBCT scanning process with a sampling period 364 of 8 seconds, during the projection process, it is necessary to 365 switch the next frame for projection every 0.2 seconds, that 366 is, each frame of data only contributes $9^{\circ}$ of projection data, 367 in other words, 40 pieces of data will provide a complete set 368 of projection data;

\section{RESULT ANALYSIS}

\section{A. Rollback reconstruction for phantom}

Firstly, we make use of a digital model to simulate the at372 tenuation of contrast agent in the process of perfusion image 373 acquisition. A circular trajectory scanner was simulated with 374 a detector to focus distance of $1250 \mathrm{~mm}$ and a source to origin 375 distance of $750 \mathrm{~mm}$. A $648 \times 474$ pixel cylindrical detector 376 was used with a maximum fan-angle of $\gamma_{\max }=0.25 \mathrm{rd}$. The 77 image matrix was $512 \times 512$ with pixels of $0.5 \times 0.5 \mathrm{~mm}^{2}$ 379 for simulation. Phantom $\mathrm{A}$ is a sphere of $8 \mathrm{~cm}$ radius, which 


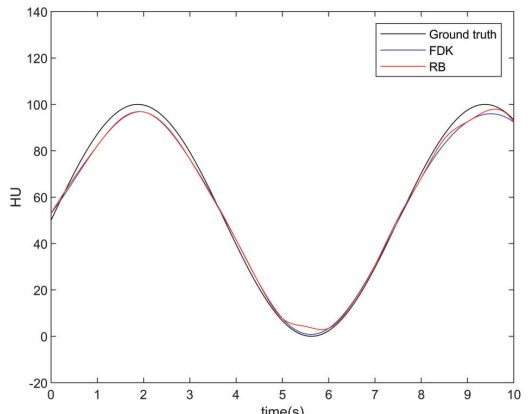

(a)

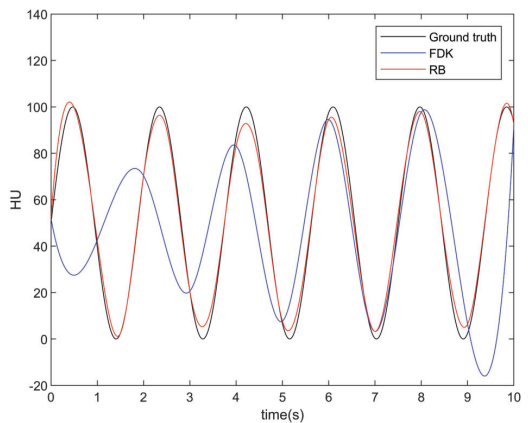

(d)

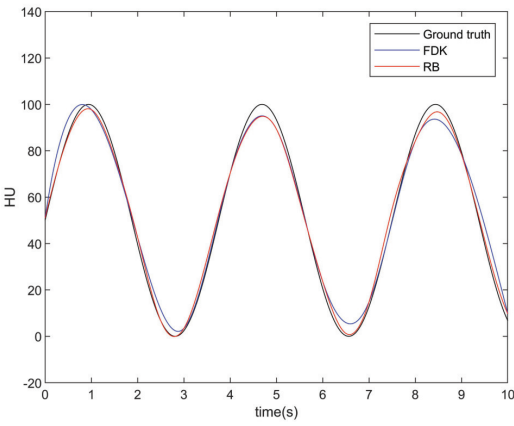

(b)

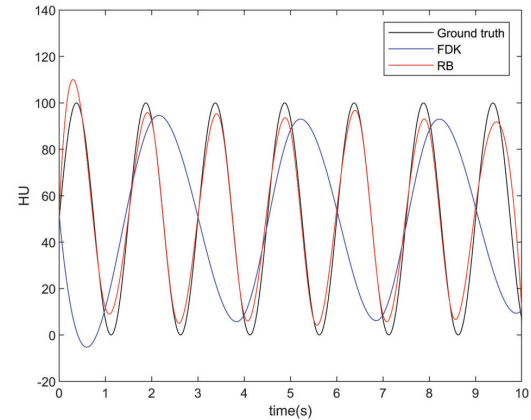

(e)

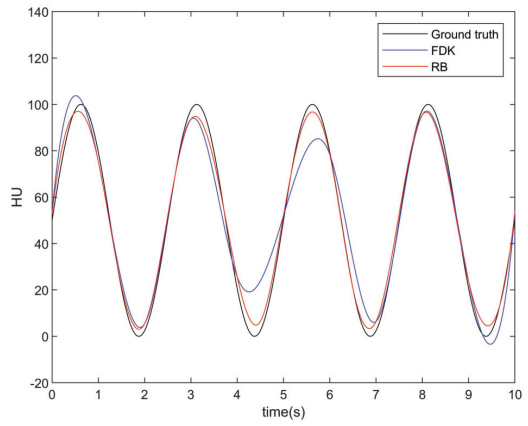

(c)

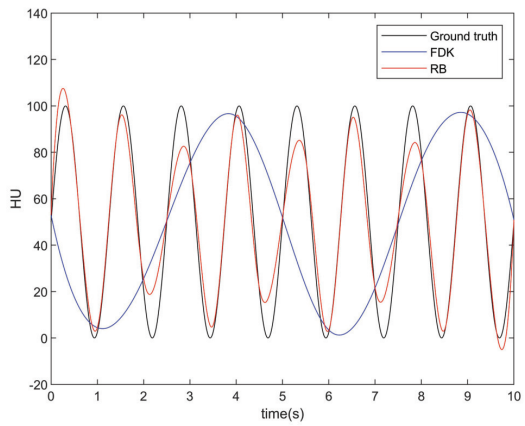

(f)

Fig. 7. The time density curves of simulated results. (a): $v_{i}=0.133$, (b): $v_{i}=0.267$, (c): $v_{i}=0.4$, (d): $v_{i}=0.534$, (e): $v_{i}=0.667$, (f): $v_{i}=0.8$
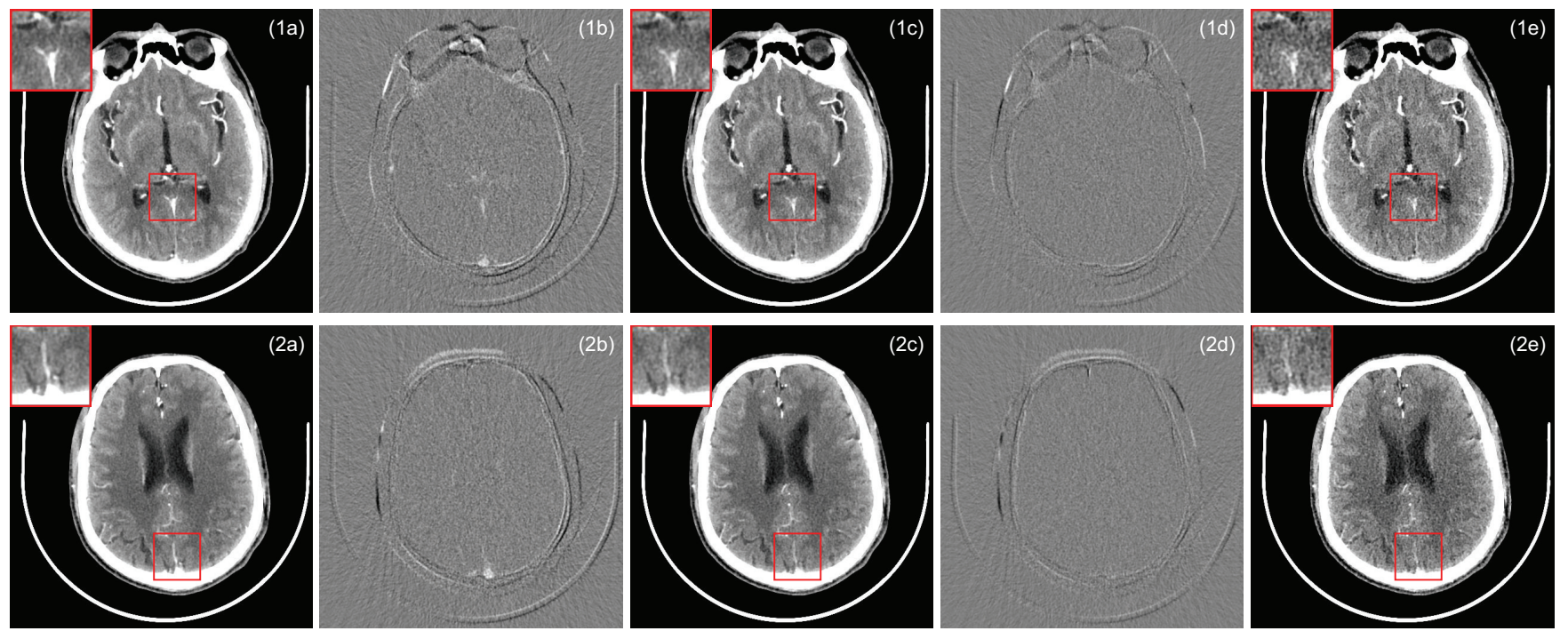

Fig. 8. (a) is the result of FDK, (b) is the error between the FDK result and the original image, (c) is the result of RBTFDK, and the result of TIA-TFDK is the same, (d) is the error between the RBTFDK result and the original image, (e) is the original image.

380 has six spherical inserts of $0.5 \mathrm{~cm}$ radius. The center of the 385 In this case, $\mu$ is the HU value, and $v$ is the frequency. ${ }_{381}$ inserts is $5.5 \mathrm{~cm}$ away from the center of the big sphere. The 386 The frequencies of the inserts are in the range $v_{i} \in[0,0.8]$. $382 \mathrm{HU}$ values of the big sphere are $50 \mathrm{HU}$ and the $\mathrm{HU}$ values of 387 Specifically, $v_{i} \in\{0.133,0.267,0.4,0.534,0.667,0.8\}$. The 383 the inserts are as follows:

388 time density curves of simulated results are presented in 389 Fig.7. They demonstrate that with the increase of frequency

$390 v$, the traditional sampling strategy is not enough to recover 


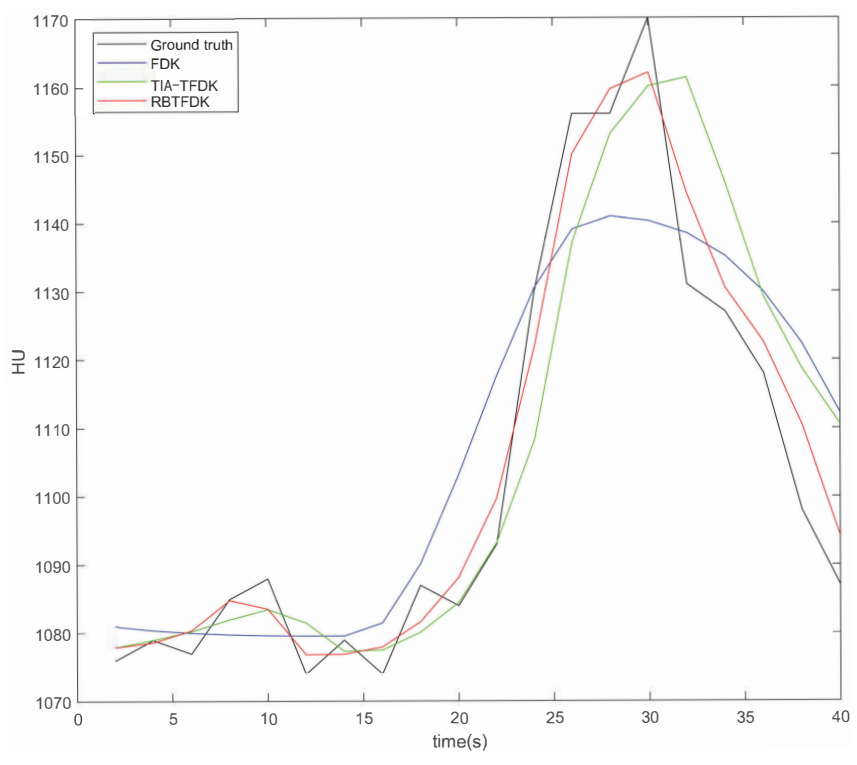

Fig. 9. Time density curves of different methods.
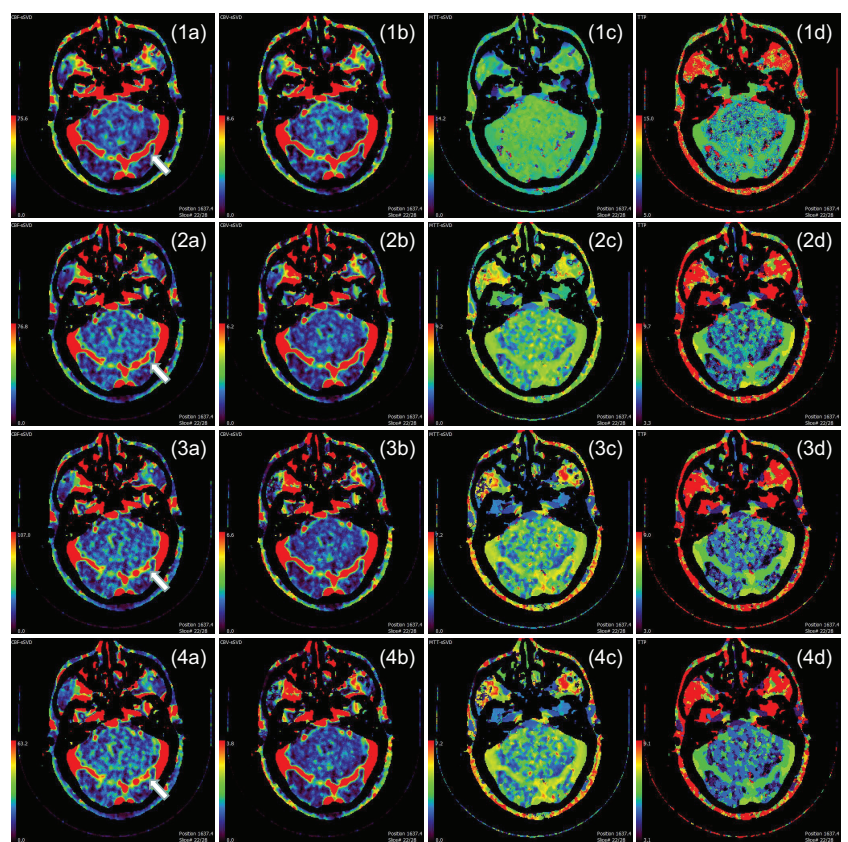

Fig. 10. Perfusion maps. From top to bottom are the results from FDK, TIA-TFDK, RBTFDK and ground truth, respectively. (a): CBF, (b): CBV, (c): MTT, (d): TTP
395

\section{B. Rollback reconstruction with TIA-TFDK(RBTFDK)}

396 The previous section has demonstrated that the rollback re397 construction could reduce the impact of time sampling prob398 lem. The rollback reconstruction is promising to tackle the 399 temporal resolution problem as well after introducing the 400 TIA-TFDK algorithm. The pre-clinical dataset was utilized 401 to validate the proposed rollback reconstruction algorithm. 402 Fig. 8 presents the results of RBTFDK method and traditional ${ }_{403}$ FDK reconstruction algorithm. The experiments demonstrate 404 that TIA-TFDK can effectively improve the reconstruction 405 accuracy and it could be used to enhance the rollback re406 construction. From Fig.9, we can observe that the rollback 407 reconstruction based on TIA-TFDK not only could preserve 408 the reconstruction accuracy but also could improve the time 409 density curve.

\section{Perfusion parameter results}

411 The perfusion maps have great significance for the diag412 nosis and treatment of stroke. Some software may overesti${ }_{413}$ mate or underestimate ischemic core. This is probably due to ${ }_{414}$ differences in tracer delay sensitivity and postprocessing al415 gorithms. The common perfusion postprocessing algorithms 416 can be classified into two categories: maximum slope and de417 convolution. Since the maximum slope method can only be 418 used to calculate CBF, it is necessary to make further assump419 tions on perfusion model when calculating CBV and other 420 perfusion parameters, so deconvolution method is often used 421 now. Kudo et al. assessed the accuracy and reliability using a 422 digital phantom with 13 perfusion postprocessing algorithms. ${ }_{423}$ Experiment results illustrated that the single value decompo424 sition(SVD) of PMA can achieve the closest CBF, CBV and 425 MTT values to the truth. Hence, PMA was employed to cal426 culate $\mathrm{CBF}$ and other perfusion maps in this study. The re427 sults in Fig.10 demonstrate that the perfusion maps obtained 428 from the RBTFDK reconstructed images match the reference 429 perfusion maps best. The RBTFDK method combined with 430 TIA-TFDK is closer to the real perfusion results in terms of 431 the details and overall appearance of perfusion images.

432 The quantitative analysis also was performed for valida433 tion. Table 1 listed the mean perfusion values of different 434 methods. The results demonstrate that the perfusion values in 435 the RBTFDK reconstructed images are well-preserved. Table ${ }_{436} 2$ and Table 3 listed the root mean square error (RMSE) and 437 mean absolute percentage error (MAPE) of different meth438 ods. Both the RMSE and MAPE show the promising perfor439 mance of the proposed RBTFDK algorithm in reconstruction 44 accuracy preservation.

\section{COMPARISON OF ALGORITHMS}

As mentioned above, The original $512 \times 512 \times 30$ data vol391 a accurate time density curve, however, the rollback recon392 struction could effectively improve the accuracy of the time 393 density curve compared with the traditional sampling strat394 egy.
446 ume for each slice location was downsampled to the dataset 448 algorithm, TIA-TFDK adds the following calculation steps: 44 of size $512 \times 512 \times 7$. Compared with the traditional FDK 
Table 1. The perfusion parameters of different methods(mean).

\begin{tabular}{lcccc}
\hline & Ground truth & FDK & TIA-FDK & RBTFDK \\
\hline CBF & 29.19 & 32.64 & 32.26 & 33.01 \\
CBV & 1.67 & 3.83 & 2.10 & 2.09 \\
MTT & 3.50 & 7.21 & 3.70 & 3.46 \\
TTP & 4.81 & 9.58 & 5.97 & 5.60 \\
\hline
\end{tabular}

Table 2. The RMSE of perfusion parameters from different methods.

\begin{tabular}{lccc}
\hline & FDK & TIA-FDK & RBTFDK \\
\hline CBF & 36.60 & 29.18 & 26.35 \\
CBV & 34.54 & 25.94 & 20.04 \\
MTT & 42.53 & 46.98 & 42.38 \\
TTP & 47.00 & 41.80 & 34.50 \\
\hline
\end{tabular}

Table 3. The MAPE of perfusion parameters from different methods.

\begin{tabular}{lccc}
\hline & FDK $(\%)$ & TIA-FDK $(\%)$ & RBTFDK $(\%)$ \\
\hline CBF & 39.23 & 32.48 & 27.65 \\
CBV & 46.50 & 29.76 & 21.99 \\
MTT & 40.22 & 32.95 & 28.64 \\
TTP & 146.60 & 76.79 & 66.67 \\
\hline
\end{tabular}
rearrangement, partial block back-projection and the in450 terpolation step. After combining rollback reconstruction, the 451 computational complexity increases. Table 4 shows the re452 construction times of the three algorithms with a PC with a 453 Intel Core i5 processor at $3.0 \mathrm{GHz}$. Indeed, the computational 454 complexity of the partial block back-projection in the TIA455 TFDK algorithm is almost negligible. The increased com456 putation time is mainly due to the interpolation step. With 457 the combination of rollback reconstruction, the computational 458 complexity increases differently according to the angle of 459 rollback. In our experiment, we take the rollback angle half 460 of the partial block back-projection. The increased time is 461 within the acceptable range.
Table 4. Reconstruction times for the three algorithms.

\begin{tabular}{cc}
\hline Algorithm & time \\
\hline FDK & $183 \mathrm{~s}$ \\
TIA-FDK & $211 \mathrm{~s}$ \\
RBTFDK & $385 \mathrm{~s}$ \\
\hline
\end{tabular}

\section{CONCLUSION}

465 In this paper, we proposed a rollback reconstruction 466 method based on TIA-TFDK algorithm for perfusion CBCT. ${ }_{467}$ In the TIA-TFDK algorithm, cone-beam projections are re468 binned to parallel-beam ones. Subsequently, the backprojec469 tion is divided in $N$ partial block backprojections. At this 470 point, we incorporate the idea of the rollback reconstruction. 471 The rollback reconstruction could tackle the time sampling 472 problem by increasing the number of sampling points. By 473 reusing the projection data that has been used for reconstruc474 tion, the number of reconstruction samples is increased with475 out additional scanning time and contrast agent dose. Then, 476 temporal interpolation is performed on the PBBs to estimate 477 the values at the desired frame times. Finally, accumulate 478 those values separately and interpolate again. In our experi479 ment, we take the partial block angle $\pi / 6$ and take the roll480 back angle half of the partial block back-projection. In fact, 481 this is the balance result between the experimental results and 482 the calculation time.

483 The algorithm was compared to the standard reconstruc484 tion and temporal interpolation approach. The experimental 485 results demonstrated that the proposed RBTFDK algorithm 486 could well preserve the accuracy of reconstructed images and 487 perfusion maps as well as improve the time density curve, 488 and show that with the decrease of partial block angle and 489 rollback angle, the reconstruction results are closer to the real 490 results. However, even from the same source data, different 491 infusion tools will get different results. This may be caused 492 by differences in contrast agent delay sensitivity and post493 processing algorithms.

494 In the future, we will focus on reducing the acquisition time 495 and radiation dose in perfusion CBCT imaging while preserv496 ing the image quality.
[1] Cho S, Pearson E, Pelizzari C A, et al. Region-of-interest image 509 reconstruction with intensity weighting in circular cone-beam 510 CT for image-guided radiation therapy[J]. Medical physics, 511 2009, 36(4): 1184-1192.

[2] Campbell B C V, Christensen S, Levi C R, et al. Cerebral blood 513 flow is the optimal CT perfusion parameter for assessing infarct 514 core[J]. Stroke, 2011, 42(12): 3435-3440.

[3] Eastwood J D, Lev M H, Wintermark M, et al. Correlation of 516 early dynamic CT perfusion imaging with whole-brain MR dif- 517 fusion and perfusion imaging in acute hemispheric stroke[J]. 518 American Journal of Neuroradiology, 2003, 24(9): 1869-1875. 519

[4] Tenjin H, Asakura F, Nakahara Y, et al. Evaluation of intraa- 520 neurysmal blood velocity by time-density curve analysis and digital subtraction angiography[J]. American journal of neuroradiology, 1998, 19(7): 1303-1307.

[5] Miles K A. Measurement of tissue perfusion by dynamic computed tomography[J]. The British journal of radiology, 1991, 64(761): 409-412.

[6] Murphy B D, Fox A J, Lee D H, et al. Identification of penumbra and infarct in acute ischemic stroke using computed tomography perfusion-derived blood flow and blood volume measurements[J]. Stroke, 2006, 37(7): 1771-1777.

[7] Miles K A, Griffiths M R. Perfusion CT: a worthwhile enhancement?[J]. The British journal of radiology, 2003, 76(904): 220- 
231.

[8] Mayer T E, Hamann G F, Baranczyk J, et al. Dynamic CT per- 548 fusion imaging of acute stroke[J]. American journal of neuro- 549 radiology, 2000, 21(8): 1441-1449.

[9] Klotz E, König M. Perfusion measurements of the brain: us- 551 ing dynamic CT for the quantitative assessment of cerebral is- 552 chemia in acute stroke[J]. European journal of radiology, 1999, 553 30(3): 170-184

[10] Fieselmann A, Ganguly A, Deuerling-Zheng Y, et al. Interven- 555 tional 4-D C-arm CT perfusion imaging using interleaved scanning and partial reconstruction interpolation[J]. IEEE transac- 557 tions on medical imaging, 2011, 31(4): 892-906.

11] Ganguly A, Fieselmann A, Marks M, et al. Cerebral CT per- 559 fusion using an interventional C-arm imaging system: cerebral 560 blood flow measurements[J]. American Journal of Neuroradi- 561 ology, 2011, 32(8): 1525-1531.

2] Montes P. Dynamic cone-beam reconstruction for perfusion 563 computed tomography[D]. , 2006.

[13] Grangeat P, Koenig A, Rodet T, et al. Theoretical framework 565 for a dynamic cone-beam reconstruction algorithm based on 566 a dynamic particle model[J]. Physics in Medicine \& Biology, 567 2002, 47(15): 2611.

14] Turbell H. Cone-beam reconstruction using filtered backpro- 560 jection[D]. Linköping University Electronic Press, 2001. tion[J]. SIAM Journal on Applied Mathematics, 1983, 43(3):
546-552.

[16] Grass M, Köhler T, Proksa R. 3D cone-beam CT reconstruction for circular trajectories[J]. Physics in Medicine \& Biology, 2000, 45(2): 329.

[17] Fieselmann A, Ganguly A, Deuerling-Zheng Y, et al. A dynamic reconstruction approach for cerebral blood flow quantification with an interventional C-arm CT[C]//2010 IEEE international symposium on biomedical imaging: from nano to macro. IEEE, 2010: 53-56.

[18] Tang J, Xu M, Niu K, et al. A novel temporal recovery technique to enable cone beam $\mathrm{CT}$ perfusion imaging using an interventional C-arm system[C]//Medical Imaging 2013: Physics of Medical Imaging. International Society for Optics and Photonics, 2013, 8668: 86681A.

[19] Li Y, Garrett J W, Li K, et al. An Enhanced SMART-RECON Algorithm for Time-Resolved C-arm Cone-Beam CT Imaging[J]. IEEE transactions on medical imaging, 2019, 39(6): 1894-1905.

[20] Zhu H, Tong D, Zhang L, et al. Temporally downsampled cerebral CT perfusion image restoration using deep residual learning[J]. International journal of computer assisted radiology and surgery, 2020, 15(2): 193-201.

[21] Montes P, Lauritsch G. A temporal interpolation approach for dynamic reconstruction in perfusion $\mathrm{CT}[\mathrm{J}]$. Medical physics, 2007, 34(7): 3077-3092. 